\title{
LISTADO DE EVALUADORES/AS 2020
}

Abreu Mendes, Irán (Universidade Federal do Pará, Brasil)

Agulló Díaz, María del Carmen (Universidad de Valencia)

Alfieri, Paolo (Università Cattolica del Sacro Cuore Milán, Italia)

Alvarez Gonzalez, Yasmina (Universidad de La Laguna)

Araújo de Oliveira, María Cristina (Universidade Federal de Juiz de Fora, Brasil) Arredondo, María Adelina (Universidad Autónoma del Estado de Morelos, México)

Barruso, Francesca (Universidad de Roma Tre, Italia)

Beas Miranda, Miguel (Universidad de Granada)

Becerra, Marina (Universidad de Buenos Aires, Argentina)

Bolaño Amigo, María Eugenia (Universidade de Santiago de Compostela)

Caldo, Paula (Universidad Nacional de Rosario)

Canales Serrano, Antonio Fco. (Universidad Complutense de Madrid)

Carrillo Márquez, Dolores (Universidad Pontificia Comillas)

Carvalho e Silva, Jaime (Universidade de Coimbra, Portugal)

Castillejo Cambra, Emilio (UNED)

Cid Castro, Eva (Universidad de Zaragoza)

Collelldemont Pujadas, Eulàlia (Universitat de Vic)

Comás Rubí, Francisca (Universitat de les Illes Balears)

Coninck-Smith, Ning (Aarhus University, Dinamarca)

Costa Rico, Antón (Universidade de Santiago de Compostela)

Cruz Orozco, José Ignacio (Universidad de Valencia)

Da Costa, Antonio (Universidade Católica de São Paulo, Brasil)

Delgado Cendagortagalarza, Ander (Universidad del País Vasco)

Delgado Granados, Patricia (Universidad de Sevilla)

Díez de Velasco Abellán, Francisco (Universidad de La Laguna)

Doval, Delfina (Universidad Nacional de Entre Ríos, Argentina)

Dussel, Inés (Centro de Investigación y de Estudios Avanzados del Instituto

Politécnico Nacional, México)

Español Gonzalez, Luis (Universidad de La Rioja)

Espín Buendía, José Ginés (Universidad de Murcia) 
Faus Prieto, Alfredo (Universidad de Valencia)

Flecha García, Consuelo (Universidad de Sevilla)

Gallego-Preciados Algora, Ana Isabel (Universidad de Castilla - La Mancha)

Garmendia Larrañaga, Joxe (Universidad del País Vasco)

Garrido de Oliveira, Carla (Universidad de Oporto, Portugal)

Gelabert Gual, Llorenç (Universitat de les Illes Balears)

Gómez Carrasco, Cosme Jesús (Universidad de Murcia)

Gonçalves Vidal, Diana Universidad de San Pablo (Brasil)

González Madrid, Damián (Universidad Castilla-La Mancha)

González Redondo, Francisco (Universidad Complutense de Madrid)

Grana Gil, Isabel (Universidad de Málaga)

Gutierrez, Laurent (Université de Picardie Jules Verne Paris, Francia)

Harford, Judith (University College Dublin, Irlanda)

Helfenberger, Marianne (Swiss University of Distance Learning, Suiza)

Kaufmann, Carolina (Universidad Nacional de Rosario, Argentina)

Kudlacova, Blanka (University of Trnava, Eslovaquia)

López Martínez, José Damián (Universidad de Valencia)

López-Ocón, Leoncio (Centro de Ciencias Humanas y Sociales del CSIC, Madrid)

Malheiro Gutiérrez, Xosé (Universidade de Santiago de Compostela)

Matos, José (Universidad de La Habana, Cuba)

Maurandi López, Antonio (Universidad de Murcia)

Mauri Medrano, Marta (Universidad de Zaragoza)

Maz Machado, Alexander (Universidad de Córdoba)

Meda, Juri (Università degli Studi di Macerata, Italia)

Monarca, Héctor (Universidad Autónoma de Madrid)

Moreno Martínez, Pedro Luis (Universidad de Murcia)

Negrín Fajardo, Olegario (UNED)

O'Doherty, Teresa (Institute of Education, Irlanda)

Olivares, Pilar (Universidad de Murcia)

Ortega Cervigón, José Ignacio (Universidad Complutense de Madrid)

Ossenbach Sauter, Gabriela (UNED)

Payá Rico, Andrés (Universidad de Valencia)

Porto Ucha, Ángel Serafín (Universidade de Santiago de Compostela)

Real Apolo, Carmelo (Universidad de Extremadura)

Ríos Zúniga, Rosalina (Universidad Nacional Autónoma de México, México)

Robles Sanjuan, Victoría (Universidad de Granada)

Rodríguez Pérez, Raimundo (Universidad de Murcia) 
Saint, Andrew (University College London, Inglaterra)

Salguero Montaño, Oscar (Universidad Complutense de Madrid)

Sánchez Botero, Clara Helena (Universidad Nacional de Bogotá, Colombia)

Sánchez Mártinez, Encarna (IES F. García Lorca, Albacete)

Sánchez-Pedreño Guillén, Salvador (Universidad de Murcia)

Sevilla Merino, Diego (Universidad de Granada)

Simon, Frank (Universiteit Gent, Bélgica)

Skiera, Ehrenhard (Universität Flensburg, Alemania)

Somogyvári, Lajos (University of Pannonia, Hungría)

Somoza Rodríguez, Miguel (UNED)

Soutelo Vazquez, Raúl (Universidad de Vigo)

Texier, Simón (Université de Picardie Jules-Verne, Francia)

Valls Montes, Rafael (Universitat Politècnica de València)

Vázquez Astorga, Mónica (Universidad de Zaragoza)

Vea Muniesa, Fernando (Universidad de Zaragoza)

Viñao Frago, Antonio (Universidad de Murcia) 